\title{
Adaptação transcultural do instrumento PAIR: Pharmacotherapy Assessment in Chronic Renal Disease para aplicação no Brasil
}

\author{
Cross-cultural adaptation of the PAIR instrument: Pharmacotherapy \\ Assessment in Chronic Renal Disease for application in Brazil
}

Alessandra Batista Marquito (https://orcid.org/0000-0003-0237-2521) ${ }^{1}$

Hélady Sanders Pinheiro (https://orcid.org/0000-0001-8603-1331) ${ }^{2}$

Rogério Baumgratz de Paula (https://orcid.org/0000-0002-7778-2907) ${ }^{2}$

${ }^{1}$ Núcleo Interdisciplinar de Estudos, Pesquisas e Tratamento em Nefrologia, Universidade Federal de Juiz de Fora (UFJF). R. José Lourenço Kelmer 1300, Centro Comercial, Bairro São Pedro. 36036-330 Juiz de Fora MG Brasil. alemarkito@yahoo.com.br ${ }^{2}$ Departamento de Clínica Médica, Faculdade de Medicina, UFJF. Juiz de Fora MG Brasil.

\begin{abstract}
Individuals suffering from chronic kidney disease (CKD) are often on multiple medications, which exposes them to drug-related problems (DRP). There are no available tools in Brazil to assess the pharmacotherapy and management of DRPs systematically in this population. The scope of this study was to perform a cross-cultural adaptation of PAIR criteria (Pharmacotherapy Assessment in Chronic Renal Disease) to Brazilian Portuguese. PAIR criteria consist of 50 DRP clinically significant items for CKD. The process of cross-cultural adaptation involved translation, synthesis, back-translation, and an analysis by an expert committee as well as a pre-test of the first draft document. A review committee consisting of 2 pharmacists and 2 nephrologists analyzed the semantic, linguistic, experiential, and conceptual equivalence between the original and translated versions. All items that obtained a score below $80 \%$ were reviewed. Word adjustments were made, as well as the exclusion of 6 DRP due to non-applicability to the Brazilian context. Thus, the final version of PAIR defined as "Avaliação da farmacoterapia na doença renal crônica" was translated and adapted into the Brazilian Portuguese language.

Key words Cross-cultural comparison, Chronic renal insufficiency, Pharmaceutical services, $\mathrm{Pa}$ tient safety
\end{abstract}

Resumo Indivíduos com doença renal crônica (DRC) frequentemente utilizam polifarmácia o que os expõe ao risco de problemas relacionados a medicamentos (PRMs). No Brasil, não existem instrumentos que possam sistematizar a avaliação da farmacoterapia e a gestão de PRMs nesta população. Portanto, o objetivo deste trabalho foi realizar a adaptação transcultural do instrumento PAIR (Pharmacotherapy Assessment in Chronic Renal Disease) para o português brasileiro. $O$ PAIR consiste numa lista de 50 PRMs considerados clinicamente significativos para renais crônicos. O processo de adaptação transcultural envolveu as etapas de tradução, síntese, retrotradução, análise por um comitê de especialistas e pré-teste da versão preliminar. O comitê de 2 farmacêuticos e 2 nefrologistas analisou a equivalência semântica, idiomática, experiencial e conceitual entre as versões original e traduzida. Todos os itens que obtiveram acordo inferior a $80 \%$ foram revisados. Foram realizados ajustes de palavras, assim como a exclusão de 6 PRMs devido à não aplicabilidade ao contexto brasileiro. Assim, foi obtida a versão final do PAIR definida como "Avaliação da farmacoterapia na doença renal crônica", traduzida e adaptada na língua portuguesa do Brasil.

Palavras-chave Comparação transcultural, Insuficiência renal crônica, Assistência farmacêutica, Segurança do paciente 


\section{Introdução}

A doença renal crônica (DRC) é caracterizada pela perda progressiva e irreversível das funções dos rins, sendo considerada um problema de saúde pública, devido à alta prevalência e a elevadas taxas de morbidade e mortalidade. No Brasil, cerca de 52 milhões de pessoas estão enquadradas na população de risco para o desenvolvimento de doenças renais por serem idosos, obesos, diabéticos, hipertensos ou por apresentarem algum histórico familiar. Além desses, 12 milhões apresentam algum grau de disfunção renal já estabelecida, cujo tratamento conservador consiste em retardar a perda da função dos rins, reduzir os sintomas e prevenir complicações ligadas à doença. Ainda mais preocupante é a constatação de que, aproximadamente, 122.000 renais crônicos em estágio avançado dependem do tratamento dialítico ou transplante para sobreviverem, a um custo de $\mathrm{R} \$ 1,4$ bilhão para os cofres públicos e privados ${ }^{1-4}$.

Esta população é constituída predominantemente por hipertensos, diabéticos e idosos, indivíduos que apresentam diversas comorbidades e que estão expostos à utilização de múltiplos medicamentos. No nosso serviço, usuários com DRC utilizam, em média, 5,6 $\pm 3,2$ medicamentos por dia, sobretudo de classes terapêuticas que atuam no sistema cardiovascular e metabólico ${ }^{5}$. A prescrição de polifarmácia, definida como uso de cinco ou mais medicamentos, faz-se necessária para o retardo da progressão da DRC e para o tratamento de suas complicações ${ }^{6}$ e de fato é relatada em $66,5 \%$ da população em tratamento ${ }^{5}$. Assim, a complexidade do regime medicamentoso aliada ao comprometimento da metabolização e excreção de fármacos pode favorecer o desencadeamento de problemas relacionados a medicamentos (PRMs) $)^{5-7}$.

O termo PRM é amplamente utilizado na literatura, porém, não há consenso sobre sua definição e classificação ${ }^{8}$. No Brasil, PRM é definido como um problema de saúde relacionado à farmacoterapia, que interfere ou pode interferir nos resultados terapêuticos e na qualidade de vida do usuário9.

Em populações de risco, como indivíduos com insuficiência cardíaca congestiva, diabetes e hipertensão arterial, os PRMs estão associados a reações adversas e erros de prescrição ${ }^{10,11}$. Em renais crônicos, há comprovação da alta prevalência de PRMs em todos os estágios da doença ${ }^{6}$, sendo que os mais comuns são o uso de medicamentos contraindicados ou em doses inade- quadas, que podem interferir de modo negativo sobre a função renal ${ }^{12}$.

Nesse contexto, um grupo de pesquisadores Canadenses desenvolveu e validou o instrumento PAIR (Pharmacotherapy Assessment in Chronic Renal Disease), que permite avaliar a farmacoterapia na DRC, com o intuito de prevenir, detectar e gerir os PRMs nesta população em tratamento conservador, isto é, na fase pré dialítica ${ }^{13}$.

O PAIR é o primeiro e único instrumento desenvolvido para farmacêuticos e profissionais de saúde que atuam em nefrologia, com foco na segurança da medicação, que permite a identificação e o manejo de PRMs de forma rápida e sistemática ${ }^{13}$. Por essa razão, essa ferramenta de suporte clínico, tem sido utilizada como referência por diversos autores, a fim de orientar, padronizar e otimizar a conduta desses profissionais ${ }^{14-18}$.

Além disso, a utilização de um registro padronizado pode gerar dados que permitem avaliar comparativamente o impacto da assistência na qualidade e na segurança do uso de medicamentos, assim como incentivar ações de melhorias entre os serviços de nefrologia ${ }^{14,16}$.

Considerando a relevância do tema e a inexistência de um instrumento específico para avaliação da farmacoterapia em renais crônicos no Brasil, o presente estudo teve como objetivo traduzir e adaptar transculturalmente o PAIR para o português, com vistas a operacionalizar a abordagem dos PRMs nesta população em nosso país.

\section{Método}

Para atingir os objetivos propostos, conduzimos estudo metodológico, que se baseia na organização e análise de dados, projetados para a avaliação e validação de instrumentos e técnicas de pesquisa ${ }^{19}$. Foi realizada a adaptação transcultural do instrumento canadense, que consiste no processo de tradução, adequação e/ou ajustes de palavras, expressões e formato, com objetivo de obter uma versão que possa ser utilizada sem prejuízos ou comprometimento dos resultados no contexto cultural brasileiro ${ }^{20}$.

A pesquisa foi desenvolvida no ambulatório de nefrologia localizado no sudeste do Brasil, que agrega um centro de Atenção Secundária em Saúde, no qual, usuários de alto risco cardiovascular, com hipertensão arterial sistêmica, diabetes mellitus e DRC, são atendidos por equipe multiprofissional, incluindo médicos de diversas especialidades, enfermeiros, nutricionistas, psicólogos, assistentes sociais, educadores físicos e 
farmacêuticos. Para a aplicação da versão preliminar do instrumento traduzido, foram incluídos prontuários de usuários com diagnóstico de DRC, em tratamento conservador, com idade igual ou superior a 18 anos e em acompanhamento no mesmo serviço por um período mínimo de 6 meses. O processo de adaptação transcultural ocorreu no período de junho de 2015 a junho de 2017.

Para que o projeto de pesquisa fosse iniciado, foi obtida a permissão dos autores do instrumento original do PAIR para o desenvolvimento da versão brasileira e aprovação do Comitê de Ética em Pesquisa da Faculdade de Ciências Médicas e da Saúde de Juiz de Fora.

\section{Instrumento de medida}

O PAIR consiste numa lista de 50 PRMs considerados clinicamente significativos para renais crônicos em tratamento conservador e que requeiram intervenção farmacêutica. Essa lista foi elaborada pelo método RAND, um procedimento que considera informações baseadas em evidências e experiência clínica de especialistas ${ }^{21}$.

Os PRMs são divididos em 6 categorias: 1 . Uso inadequado do medicamento devido prescrição inapropriada (dose incorreta ou medicamento contra-indicado); 2 . Aderência inadequada ao tratamento; 3 . Pressão arterial inadequada; 4. Hipoglicemia secundária à sulfoniluréias; 5 . Interação medicamentosa e situações em que o medicamento é tomado inadequadamente; 6. Problemas relacionados com medicamentos isentos de prescrição médica ou produtos naturais de saúde ${ }^{13}$.

Assim, o uso desse instrumento permite avaliar a terapia farmacológica instituída para o renal crônico, sendo importante para otimizar os resultados clínicos esperados pelo médico.

\section{Procedimento de adaptação transcultural}

O procedimento de adaptação transcultural foi realizado seguindo recomendações metodológicas internacionais ${ }^{20,22-25}$ na sequência apresentada a seguir e na Figura 1.

a) Tradução inicial: esta etapa consistiu na realização independente de duas traduções do instrumento da língua original (inglês) para a língua alvo (português), por dois tradutores bilíngues, cuja língua materna era portuguesa (T1 e T2).

b) Síntese das traduções: uma terceira pessoa imparcial produziu a síntese das duas traduções, resultando em uma tradução comum (T-12). c) Retrotradução: a versão T-12 do instrumento foi traduzida de volta para a língua original (inglês) por duas pessoas bilingues com o idioma de origem (inglês) como sua língua materna (BT1 e BT2). As retrotraduções foram sintetizadas e o resultado confrontado com o original para correção de eventuais discordâncias.

d) Análise por um comitê de especialistas: um comitê de especialistas composto por 2 médicos nefrologistas e 2 farmacêuticos clínicos, todos com proficiência em língua inglesa, foi convocado para produzir a versão preliminar do PAIR traduzido (consolidação das versões T1, T2, T12, BT1, BT2) e analisá-lo quanto às equivalências semântica (as palavras terem o mesmo significado); idiomática (coloquialismos ou expressões idiomáticas); experiencial (situações cotidianas descritas na versão original devem adaptar-se ao contexto da cultura alvo em que se realiza); conceitual (solucionar significado conceitual diferente entre as culturas). Para essa etapa foram fornecidos aos membros do comitê individualmente os instrumentos na forma original e traduzida, um roteiro de orientações e um instrumento de avaliação. O envio e devoluções dos documentos foram realizados através de correio eletrônico. A cada item foi solicitado um conceito $(-1=$ não equivale; $0=$ indeciso $;+1=$ equivalente $)$. Nos casos em que foram atribuídos os conceitos - 1 e 0 , o especialista deveria fazer um comentário/ sugestão. Após a entrega de todas as avaliações, o grau de concordância entre os especialistas foi mensurado. Todos os itens que obtiveram níveis de concordância inferior a $80 \%$ foram revisados, e aqueles com nível de concordância igual ou inferior a $50 \%$ discutidos presencialmente a fim de se obter um consenso.

e) Pré-teste: etapa realizada a fim de verificar a compreensão e aplicabilidade da segunda versão do PAIR revisada. Foram convidados a participar dessa etapa dois farmacêuticos e um médico com mesmo nível de conhecimento em nefrologia (profissionais inseridos em serviço de atendimento multiprofissional, que participam rotineiramente de grupos de discussão e atualização em DRC). Após serem devidamente instruídos, os 3 profissionais selecionados aplicaram o instrumento de forma independe em 10 prontuários, que foram sorteados aleatoriamente e que atenderam aos critérios de inclusão, a fim de identificar qualquer dificuldade prática. Todas as sugestões propostas nessa etapa foram analisadas, de forma conjunta e criticamente, por profissionais envolvidos nas etapas anteriores, incluindo a pesquisadora responsável pelo 


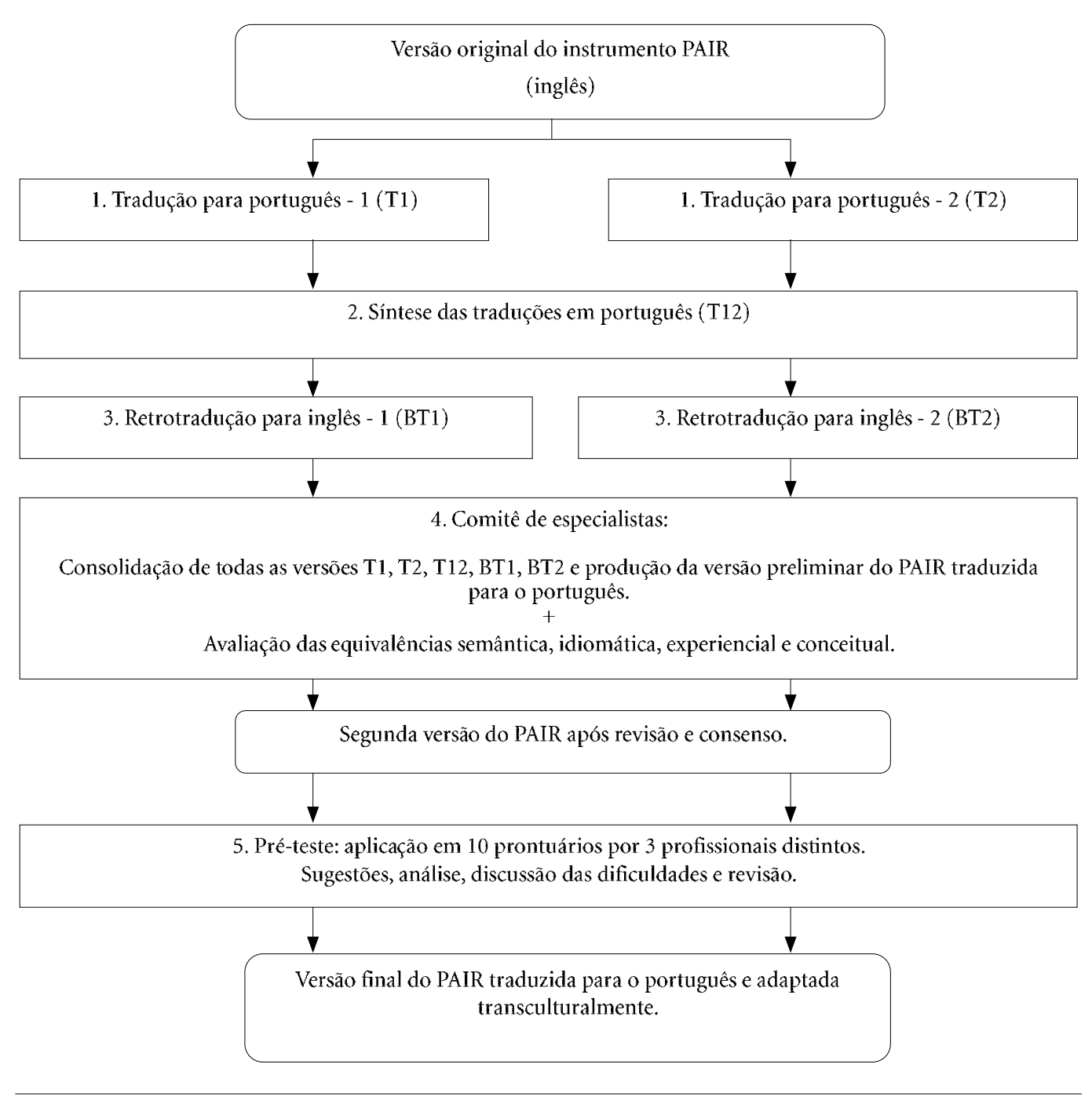

Figura 1. Fluxograma das etapas realizadas para o processo de adaptação transcultural do instrumento PAIR. Juiz de Fora-MG, Brasil, 2017.

trabalho e um especialista em adaptação transcultural de métodos. Nessa etapa também foram esclarecidas dúvidas com relação à interpretação do texto com os autores do trabalho original via correio eletrônico. A análise descritiva dos dados foi realizada com o Statistical Package for the Social Sciences 15.0 (SPSS Inc., Chicago, IL, USA). Obteve-se assim, por consenso, a versão final do PAIR traduzida e adaptada transculturalmente.

\section{Resultados}

O Quadro 1 apresenta o resultado da adaptação transcultural do PAIR. A primeira coluna apresenta os itens da versão original em inglês. A segunda coluna apresenta a versão final em português, após as modificações realizadas pela comissão de especialistas, com base na análise das traduções e retrotraduções, nas sugestões dadas pelos profissionais de saúde do ambulatório, nas dúvidas enviadas e esclarecidas com os autores do instrumento original e nas informações obtidas após o pré-teste.

Os estágios de tradução, síntese e retrotradução apresentaram 13 discrepâncias, com nível de concordância igual a 50\%, que foram discutidas e resolvidas por consenso entre os tradutores. Oito alterações foram feitas pelo comitê de especialistas na redação e cinco PRMs da categoria 
Quadro 1. Resultado do processo de adaptação transcultural do instrumento PAIR (Pharmacotherapy Assessment in Chronic Renal Disease).

\begin{tabular}{|c|c|}
\hline Versão original em Inglês & Versão final traduzida e adaptada em Português \\
\hline $\begin{array}{c}\text { Pharmacotherapy assessment in chronic renal } \\
\text { disease }\end{array}$ & Avaliação da farmacoterapia na doença renal crônica \\
\hline $\begin{array}{l}\text { Inadequate use (inappropriate dosage or contra- } \\
\text { indicated agent) }\end{array}$ & $\begin{array}{l}\text { Uso inadequado (dose inapropriada ou } \\
\text { medicamento contraindicado) }\end{array}$ \\
\hline $\begin{array}{l}\text { 1. The patient is receiving a medication that is not } \\
\text { indicated, a non-steroidal anti-inflammatory. }\end{array}$ & $\begin{array}{l}\text { 1. O paciente está recebendo um medicamento } \\
\text { contraindicado, um anti-inflamatório não esteroidal. }\end{array}$ \\
\hline
\end{tabular}

2. The patient is receiving too high a dose of gabapentin.

2. O paciente está recebendo uma dose muito elevada de gabapentina:

CrCl 30-59 mL/min: maximum dose $1400 \mathrm{mg} /$ day P.O. TFG 30-59 mL/min: dose máxima de $1400 \mathrm{mg} / \mathrm{dia}$, via $\mathrm{CrCl} 15-29 \mathrm{~mL} / \mathrm{min}$ : maximum dose $700 \mathrm{mg} /$ day P.O. oral;

$\mathrm{CrCl}$ 10-14 mL/min: maximum dose $300 \mathrm{mg} /$ day P.O. TFG $15-29 \mathrm{~mL} / \mathrm{min}$ : dose máxima de $700 \mathrm{mg} /$ dia, via $\mathrm{CrCl}<10 \mathrm{~mL} / \mathrm{min}$ : maximum dose $150 \mathrm{mg}$ /day P.O.

oral;

TFG 10-14 mL/min: dose máxima de $300 \mathrm{mg} / \mathrm{dia}$, via oral;

TFG $<10 \mathrm{~mL} / \mathrm{min}$ : dose máxima de $150 \mathrm{mg} / \mathrm{dia}$, via oral.

3. The patient is receiving a medication that is not indicated, meperidine.

3. O paciente está recebendo um medicamento contraindicado, meperidina.

4. The patient is receiving too high a dose of pregabalin.

4. O paciente está recebendo uma dose muito elevada de pregabalina.

$\mathrm{CrCl}$ 30-59 mL/min: maximum dose $300 \mathrm{mg} /$ day P.O. $\mathrm{CrCl} 15-29 \mathrm{~mL} / \mathrm{min}$ : maximum dose $150 \mathrm{mg} /$ day P.O. $\mathrm{CrCl}<15 \mathrm{~mL} / \mathrm{min}$ : maximum dose $75 \mathrm{mg} /$ day P.O.

5. The patient is receiving too high a dose of an antiviral (acyclovir, valacyclovir, famciclovir) according to the dosage-adjustment tables for kidney disease.

6. The patient is receiving too high a dose of cephalosporin according to the dosage-adjustment tables for kidney disease.

7. The patient is receiving too high a dose of a neuraminidase inhibitor (e.g., oseltamivir) according to the dosage-adjustment tables for kidney disease.

TFG 30-59 mL/min: dose máxima de $300 \mathrm{mg} /$ dia, via oral; TFG $15-29 \mathrm{~mL} / \mathrm{min}$ : dose máxima de $150 \mathrm{mg} / \mathrm{dia}$, via oral; TFG $<15 \mathrm{~mL} / \mathrm{min}$ : dose máxima de $75 \mathrm{mg} / \mathrm{dia}$, via oral.

5. O paciente está recebendo uma dose muito elevada de um antiviral (aciclovir, valaciclovir, fanciclovir), de acordo com as tabelas de ajuste de dose para doença renal.

6. O paciente está recebendo uma dose muito elevada de cefalosporina, de acordo com as tabelas de ajuste de dose para doença renal.

7. O paciente está recebendo uma dose muito elevada de inibidor da neuraminidase (oseltamivir, por exemplo), de acordo com as tabelas de ajuste de dose para doença renal.

8. The patient is receiving nitrofurantoin, which is 8. O paciente está recebendo nitrofurantoína, a qual é contra-indicated in kidney disease $(\mathrm{CrCl}<60 \mathrm{~mL} /$ $\min )$. contraindicada na doença renal (TFG $<60 \mathrm{~mL} / \mathrm{min}$ ).

9. The patient is receiving too high a dose of penicillin according to the dosage-adjustment tables for kidney disease.

10. The patient is receiving too high a dose of a quinolone according to the dosage-adjustment tables for kidney disease.

11. The patient is receiving too high a dose of a sulfamide according to the dosage-adjustment tables for kidney disease.

12. The patient is receiving too high a dose of a tetracycline according to the dosage-adjustment tables for kidney disease.

13. The patient is receiving too high a dose of a triazole (e.g., fluconazole) according to the dosageadjustment tables for kidney disease.

9. O paciente está recebendo uma dose muito elevada de penicilina, de acordo com as tabelas de ajuste de dose para doença renal.

10. O paciente está recebendo uma dose muito elevada de uma quinolona, de acordo com as tabelas de ajuste de dose para doença renal.

11. O paciente está recebendo uma dose muito elevada de uma sulfonamida, de acordo com as tabelas de ajuste de dose para doença renal.

12. O paciente está recebendo uma dose muito elevada de uma tetraciclina, de acordo com as tabelas de ajuste de dose para doença renal.

13. O paciente está recebendo uma dose muito elevada de um triazólico (fluconazol, por exemplo), de acordo com as tabelas de ajuste de dose para doença renal. 
Quadro 1. Resultado do processo de adaptação transcultural do instrumento PAIR (Pharmacotherapy Assessment in Chronic Renal Disease).

\begin{tabular}{|c|c|}
\hline $\begin{array}{l}\text { 14. The patient is receiving too high a dose of a beta } \\
\text { blocker according to the dosage-adjustment tables for } \\
\text { kidney disease. }\end{array}$ & $\begin{array}{l}\text { 14. O paciente está recebendo uma dose muito elevada } \\
\text { de um betabloqueador, de acordo com as tabelas de } \\
\text { ajuste de dose para doença renal. }\end{array}$ \\
\hline $\begin{array}{l}\text { 15. The patient is receiving too high a dose of } \\
\text { fenofibrate nanocrystals } \\
\mathrm{CrCl} 20-50 \mathrm{~mL} / \mathrm{min} \text { : initial maximum dose } 48 \mathrm{mg} / \\
\text { day P.O. }\end{array}$ & $\begin{array}{l}\text { 15. O paciente está recebendo uma dose muito elevada } \\
\text { de nanocristais de fenofibrato. } \\
\text { TFG } 20-50 \mathrm{~mL} / \mathrm{min} \text { : dose máxima inicial de } 48 \mathrm{mg} / \\
\text { dia, via oral. }\end{array}$ \\
\hline $\begin{array}{l}\text { 16. The patient has a } \mathrm{CrCl}<25 \mathrm{~mL} / \mathrm{min} \text { and is } \\
\text { receiving a medication that is not indicated, acarbose. }\end{array}$ & $\begin{array}{l}\text { 16. O paciente apresenta uma TFG }<25 \mathrm{~mL} / \mathrm{min} \text { e } \\
\text { está recebendo um medicamento contraindicado, } \\
\text { acarbose. }\end{array}$ \\
\hline $\begin{array}{l}\text { 17. The patient has a } \mathrm{CrCl}<30 \mathrm{~mL} / \mathrm{min} \text { and is taking } \\
\text { a medication that is not indicated, metformin. }\end{array}$ & $\begin{array}{l}\text { 17. O paciente apresenta uma } \mathrm{TFG}<30 \mathrm{~mL} / \mathrm{min} \\
\text { e está tomando um medicamento contraindicado, } \\
\text { metformina. }\end{array}$ \\
\hline $\begin{array}{l}\text { 18. The patient is receiving too high a dose of } \\
\text { ranitidine. } \\
\mathrm{CrCl}<50 \mathrm{~mL} / \mathrm{min} \text { : maximum dose } 150 \mathrm{mg} / \text { day P.O. } \\
\text { If needed may be increased to } 150 \mathrm{mg} / \text { day bid if } \mathrm{CrCl} \\
\text { is between } 30 \text { and } 50 \mathrm{~mL} / \mathrm{min} \text {. }\end{array}$ & $\begin{array}{l}\text { 18. O paciente está recebendo uma dose muito elevada } \\
\text { de ranitidina. } \\
\text { TFG }<50 \mathrm{~mL} / \mathrm{min} \text { : dose máxima de } 150 \mathrm{mg} / \text { dia, via oral. } \\
\text { Se necessário, pode ser aumentada para } 150 \mathrm{mg} / \text { dia, duas } \\
\text { vezes ao dia, se a TFG estiver entre } 30 \text { e } 50 \mathrm{~mL} / \mathrm{min} \text {. }\end{array}$ \\
\hline $\begin{array}{l}\text { 19. The patient is receiving too high a dose of } \\
\text { allopurinol. } \\
\mathrm{CrCl} 41-60 \mathrm{~mL} / \mathrm{min} \text { : maximum dose } 150 \mathrm{mg} \text { P.O. DIE } \\
\mathrm{CrCl} 21-40 \mathrm{~mL} / \mathrm{min} \text { : maximum dose } 100 \mathrm{mg} \text { P.O. DIE } \\
\mathrm{CrCl} 10-20 \mathrm{~mL} / \mathrm{min} \text { : maximum dose } 100 \mathrm{mg} \text { P.O. } \\
\text { every second day } \\
\mathrm{CrCl}<10 \mathrm{~mL} / \mathrm{min} \text { : maximum dose } 100 \mathrm{mg} \text { P.O. every } \\
3 \text { days }\end{array}$ & $\begin{array}{l}\text { 19. O paciente está recebendo uma dose muito elevada de } \\
\text { alopurinol. } \\
\text { TFG } 41-60 \mathrm{~mL} / \mathrm{min} \text { : dose máxima de } 150 \mathrm{mg} / \text { dia, via } \\
\text { oral, uma vez ao dia; } \\
\text { TFG } 21-40 \mathrm{~mL} / \mathrm{min} \text { : dose máxima de } 100 \mathrm{mg} / \mathrm{dia} \text {, via } \\
\text { oral, uma vez ao dia; } \\
\text { TFG } 10-20 \mathrm{~mL} / \mathrm{min} \text { : dose máxima de } 100 \mathrm{mg} / \mathrm{dia} \text {, via } \\
\text { oral, em dias alternados; } \\
\text { TFG < } 10 \mathrm{~mL} / \mathrm{min} \text { : dose máxima de } 100 \mathrm{mg} / \mathrm{dia} \text {, via oral, } \\
\text { a cada três dias. }\end{array}$ \\
\hline $\begin{array}{l}\text { 20. The patient is receiving too high a dose of } \\
\text { colchicine as a prophylactic treatment for gout: } \\
\mathrm{CrCl}<50 \mathrm{~mL} / \mathrm{min} \text { : not recommended for chronic } \\
\text { treatment. } \\
\text { The patient is receiving a too high dose of colchicine } \\
\text { for an acute treatment: } \\
\mathrm{CrCl} 35-50 \mathrm{~mL} / \mathrm{min} \text { : maximum dose } 0.6 \mathrm{mg} / \text { day } \\
\mathrm{CrCl}<35 \mathrm{~mL} / \mathrm{min} \text { : maximum dose } 0.3 \mathrm{mg} \text { P.O. DIE } \\
\text { or } 0.6 \mathrm{mg} \text { P.O. every } 2 \text { days }\end{array}$ & $\begin{array}{l}\text { 20. O paciente está recebendo uma dose muito elevada } \\
\text { de colchicina, como tratamento profilático para gota: } \\
\text { TFG }<50 \mathrm{~mL} / \mathrm{min} \text { : tratamento crônico não } \\
\text { recomendado. } \\
\text { O paciente está recebendo uma dose muito elevada de } \\
\text { colchicina para um tratamento agudo: } \\
\text { TFG } 35-50 \mathrm{~mL} / \mathrm{min} \text { : dose máxima de } 0,6 \mathrm{mg} / \mathrm{dia} \text {; } \\
\text { TFG }<35 \mathrm{~mL} / \mathrm{min} \text { : dose máxima de } 0,3 \mathrm{mg} \text {, via } \\
\text { oral, uma vez ao dia, ou } 0,6 \mathrm{mg} \text {, via oral, em dias } \\
\text { alternados. }\end{array}$ \\
\hline $\begin{array}{l}\text { 21. The patient with a } \mathrm{CrCl}<30 \mathrm{~mL} / \mathrm{min} \text { is taking a } \\
\text { medication that is not indicated, a bisphosphonate } \\
\text { (alendronate, etidronate, risedronate). }\end{array}$ & $\begin{array}{l}\text { 21. O paciente com uma TFG }<30 \mathrm{~mL} / \mathrm{min} \text { está } \\
\text { tomando um medicamento contraindicado, um } \\
\text { bifosfonato (alendronato, etidronato, risedronato). }\end{array}$ \\
\hline $\begin{array}{l}\text { 22. The patient is receiving too high a dose of } \\
\text { varenicline. } \\
\mathrm{CrCl}<10-30 \mathrm{~mL} / \mathrm{min} \text { : maximum dose } 0.5 \mathrm{mg} \text { P.O. } \\
\mathrm{BID} \\
\mathrm{CrCl}<10 \mathrm{~mL} / \mathrm{min} \text { : maximum dose } 0.5 \mathrm{mg} \text { P.O. DIE }\end{array}$ & $\begin{array}{l}\text { 22. O paciente está recebendo uma dose muito elevada } \\
\text { de vareniclina. } \\
\text { TFG } 10-30 \mathrm{~mL} / \mathrm{min} \text { : dose máxima de } 0,5 \mathrm{mg} \text {, via oral, } \\
\text { duas vezes ao dia; } \\
\text { TFG < } 10 \mathrm{~mL} / \mathrm{min} \text { : dose máxima de } 0,5 \mathrm{mg} \text {, via oral, } \\
\text { uma vez ao dia. }\end{array}$ \\
\hline
\end{tabular}

continua

"Aderência Inadequada" foram rejeitados porque não foram considerados aplicáveis ao Sistema de Saúde do Brasil por se tratarem de controle de dispensação de medicamentos.
Com relação às características dos pacientes dos 10 prontuários escolhidos aleatoriamente para a avaliação no pré-teste, $60 \%$ eram do sexo feminino, apresentando média de idade de 68 
Quadro 1. Resultado do processo de adaptação transcultural do instrumento PAIR (Pharmacotherapy Assessment in Chronic Renal Disease).

\begin{tabular}{|c|c|}
\hline Non-optimal adherence & Aderência Inadequada \\
\hline $\begin{array}{l}\text { 23. The patient is non-adherent to his anemia } \\
\text { treatment since he received more than } 120 \% \text { of the } \\
\text { required quantity of drug over } 90 \text { consecutive days or } \\
\text { less than } 80 \% \text { of the required quantity of drug over } 90 \\
\text { consecutive days. }\end{array}$ & Categoria excluída \\
\hline 24. The patient is non-adherent to his antihypertensive & \\
\hline
\end{tabular}

treatment since he received more than $120 \%$ of the required quantity of drug over 90 consecutive days or less than $80 \%$ of the required quantity of drug over 90 consecutive days.

25. The patient is non-adherent to his hypolipidemic treatment since he received more than $120 \%$ of the required quantity of drug over 90 consecutive days or less than $80 \%$ of the required quantity of drug over 90 consecutive days.

26. The patient is non-adherent to his treatment to regulate phosphocalcic metabolism since he received more than $120 \%$ of the required quantity of drug over 90 consecutive days or less than $80 \%$ of the required quantity of drug over 90 consecutive days.

27. The patient is non-adherent to his diabetes treatment since he received more than $120 \%$ of the required quantity of drug over 90 consecutive days or less than $80 \%$ of the required quantity of drug over 90 consecutive days.

\begin{tabular}{|c|}
\hline Non-optimal blood pressure \\
\hline 28 . The patient needs drug treatment since his blood
\end{tabular}
pressure $>130 / 80 \mathrm{~mm} \mathrm{Hg}$, but he is not receiving it.

23. O paciente precisa de tratamento medicamentoso, pois a sua pressão sanguínea está $>130 / 80 \mathrm{mmHg}$, mas ele não está recebendo-o.

29. The patient is receiving too low a dose of his antihypertensive agent since his blood pressure > 130/80 mm Hg.

\begin{tabular}{l} 
Hypoglycaemia secondary to sulfonylurea \\
\hline $\begin{array}{l}\text { 30. The patient is experiencing an adverse reaction } \\
\text { (hypoglycaemia) after taking his oral hypoglycaemic } \\
\text { drug (first generation sulfonylurea: chlorpropamide, } \\
\text { tolbutamide) }\end{array}$
\end{tabular}

31. The patient is experiencing an adverse reaction (hypoglycaemia) after taking his oral hypoglycaemic drug (second generation sulfonylurea: glyburide)

24. O paciente está recebendo uma dose muito baixa do seu anti-hipertensivo, e, por conseguinte, a sua pressão sanguínea está $>130 / 80 \mathrm{mmHg}$. Hipoglicemia secundária à sulfoniluréia PRM excluído

Interaction and drug taken inadequately

32. The patient is experiencing a drug interaction between calcium carbonate and an antibiotic (tetracycline or fluoroquinolone except moxifloxacin).

33. The patient is experiencing a drug interaction between calcium and iron P.O. taken concomitantly.

25. O paciente está desenvolvendo uma reação adversa (hipoglicemia) após tomar o seu medicamento hipoglicemiante oral (sulfoniluréia de segunda geração: glibenclamida).

Interação e medicamento tomado inadequadamente

26. O paciente está desenvolvendo uma interação medicamentosa entre carbonato de cálcio e um . antibiótico (tetraciclina ou fluoroquinolona, exceto moxifloxacino).

27. O paciente está desenvolvendo uma interação medicamentosa entre cálcio e ferro tomados concomitantemente, por via oral.

34. The patient is not taking his phosphate binder (calcium carbonate, sevelamer or lanthanum) 28. O paciente não está tomando o seu quelante de fósforo (carbonato de cálcio, acetato de cálcio, sevelâmer ou lantânio) apropriadamente. 
Quadro 1. Resultado do processo de adaptação transcultural do instrumento PAIR (Pharmacotherapy Assessment in Chronic Renal Disease).

\begin{tabular}{|c|c|}
\hline $\begin{array}{l}\text { 35. The patient is experiencing a drug interaction } \\
\text { between his phosphate binder (calcium carbonate, } \\
\text { sevelamer or lanthanum and levothyroxine). }\end{array}$ & $\begin{array}{l}\text { 29. O paciente está desenvolvendo uma interação } \\
\text { medicamentosa entre o seu quelante de fósforo } \\
\text { (carbonato de cálcio, acetato de cálcio, sevelâmer ou } \\
\text { lantânio e levotiroxina). }\end{array}$ \\
\hline $\begin{array}{l}\text { 36. The patient is experiencing a drug interaction } \\
\text { between sevelamer or lanthanum and ciprofloxacin. }\end{array}$ & $\begin{array}{l}\text { 30. O paciente está desenvolvendo uma interação } \\
\text { medicamentosa entre sevelâmer ou lantânio, e } \\
\text { ciprofloxacino. }\end{array}$ \\
\hline $\begin{array}{l}\text { 37. The patient is not taking his vitamin } \mathrm{D} \text { (calcitriol } \\
\text { or alfacalcidol) appropriately. }\end{array}$ & $\begin{array}{l}\text { 31. O paciente não está tomando a sua vitamina D } \\
\text { (calcitriol ou alfacalcidol) apropriadamente. }\end{array}$ \\
\hline $\begin{array}{l}\text { 38. The patient is not taking his sodium polystyrene } \\
\text { sulfonate appropriately. }\end{array}$ & $\begin{array}{l}\text { 32. O paciente não está tomando o seu } \\
\text { poliestirenossulfonato de cálcio apropriadamente. }\end{array}$ \\
\hline $\begin{array}{l}\text { 39. The patient needs a referral for smoking cessation } \\
\text { treatment or follow-up but has not received it. }\end{array}$ & $\begin{array}{l}\text { 33. O paciente necessita ser encaminhado para } \\
\text { tratamento ou acompanhamento da cessação do } \\
\text { tabagismo, mas não o tem recebido. }\end{array}$ \\
\hline $\begin{array}{c}\text { Problems related to an over-the-counter medication } \\
\text { or a natural health product }\end{array}$ & $\begin{array}{c}\text { Problemas relacionados com medicamento isento de } \\
\text { prescrição médica ou produto natural de saúde }\end{array}$ \\
\hline $\begin{array}{l}\text { 40. The patient is taking medication not indicated for } \\
\text { chronic kidney disease, an antacid containing calcium, } \\
\text { magnesium, aluminum and/or sodium. }\end{array}$ & $\begin{array}{l}\text { 34. O paciente está tomando medicamento } \\
\text { contraindicado para doença renal crônica, um antiácido } \\
\text { contendo cálcio, magnésio, alumínio e/ou sódio. }\end{array}$ \\
\hline $\begin{array}{l}\text { 41. The patient is experiencing an adverse reaction } \\
\text { (hypertension) to pseudoephedrine or phenyleprhine. }\end{array}$ & $\begin{array}{l}\text { 35. O paciente está desenvolvendo uma reação adversa } \\
\text { (hipertensão) a pseudoefedrina ou a fenilefrina. }\end{array}$ \\
\hline $\begin{array}{l}\text { 42. The patient is taking a purgative not indicated for } \\
\text { kidney. }\end{array}$ & $\begin{array}{l}\text { 36. O paciente está tomando um laxante } \\
\text { contraindicado para o rim. }\end{array}$ \\
\hline $\begin{array}{l}\text { 43. The patient is receiving a medication not indicated } \\
\text { for a patient with kidney disease, a vitamin A enriched } \\
\text { multivitamin. }\end{array}$ & $\begin{array}{l}\text { 37. O paciente está recebendo um medicamento } \\
\text { contraindicado para paciente com doença renal, uma } \\
\text { multivitamina enriquecida com vitamina A. }\end{array}$ \\
\hline $\begin{array}{l}\text { 44. The patient with chronic kidney disease is } \\
\text { receiving too high a dose of an ascorbic acid } \\
\text { supplement (vitamin C), a dose }>250 \mathrm{mg} / \text { day. }\end{array}$ & $\begin{array}{l}\text { 38. O paciente com doença renal crônica está } \\
\text { recebendo uma dose muito elevada de um suplemento } \\
\text { de ácido ascórbico (vitamina } \mathrm{C} \text { ) }>250 \mathrm{mg} / \text { dia. }\end{array}$ \\
\hline $\begin{array}{l}\text { 45. The patient is receiving a natural product not } \\
\text { indicated in kidney disease, a garlic supplement. }\end{array}$ & $\begin{array}{l}\text { 39. O paciente está recebendo um produto natural } \\
\text { contraindicado na doença renal, um suplemento de } \\
\text { alho. }\end{array}$ \\
\hline $\begin{array}{l}\text { 46. The patient is receiving a natural product not } \\
\text { indicated for transplant patients with kidney disease, } \\
\text { echinacea. }\end{array}$ & $\begin{array}{l}\text { 40. O paciente está recebendo um produto natural } \\
\text { contraindicado para pacientes transplantados com } \\
\text { doença renal, equinácea. }\end{array}$ \\
\hline $\begin{array}{l}\text { 47. The patient is receiving a natural product not } \\
\text { indicated in kidney disease, ginkgo biloba. }\end{array}$ & $\begin{array}{l}\text { 41. O paciente está recebendo um produto natural } \\
\text { contraindicado na doença renal, ginkgo biloba. }\end{array}$ \\
\hline $\begin{array}{l}\text { 48. The patient is receiving a natural product not } \\
\text { indicated for transplant patients, cat's claw. }\end{array}$ & $\begin{array}{l}\text { 42. O paciente está recebendo um produto natural } \\
\text { contraindicado para pacientes transplantados, unha } \\
\text { de gato. }\end{array}$ \\
\hline $\begin{array}{l}\text { 49. The patient is receiving a natural product not } \\
\text { indicated in kidney disease, St. John's wort. }\end{array}$ & $\begin{array}{l}\text { 43. O paciente está recebendo um produto natural } \\
\text { contraindicado na doença renal, erva de São João. }\end{array}$ \\
\hline $\begin{array}{l}\text { 50. The patient is receiving a natural product not } \\
\text { indicated in kidney disease, liquorice root. }\end{array}$ & $\begin{array}{l}\text { 44. O paciente está recebendo um produto natural } \\
\text { contraindicado na doença renal, raiz de alcaçuz. }\end{array}$ \\
\hline $\begin{array}{l}{ }^{*} \text { Abbreviations: BID, twice a day; } \mathrm{CrCl} \text {, creatinine } \\
\text { clearance; DIE, once a day; DRPs, drug-related } \\
\text { problems; IV, intravenous; P.O., orally. }\end{array}$ & $\begin{array}{l}\text { *Abreviações: TFG: taxa de filtração glomerular; PRM: } \\
\text { problema relacionado com o medicamento. }\end{array}$ \\
\hline
\end{tabular}

anos, 60\% no estágio intermediário da DRC (estágio 3B), 80\% concomitantemente diabéticos e hipertensos, utilizando uma média de 8,2 medicamentos.
Durante a fase de pré-teste, o PAIR mostrouse um instrumento de fácil aplicação, com duração média de aplicação de 15 minutos. Foram realizados apenas 3 ajustes. Mais um PRM, da 
categoria "Hipoglicemia secundária à sulfoniluréia”, foi rejeitado. Houve dificuldade de compreender o objetivo do termo "não tomar o medicamento apropriadamente" utilizado no artigo original nos PRMs numerados como 34, 37 e 38 . Dessa forma, foi necessário esclarecer as dúvidas diretamente com a autora nessa etapa do processo, para que a fidedignidade do instrumento fosse mantida. Após esses ajustes, a versão final adaptada ao Brasil, foi gerada.

\section{Discussão}

Este trabalho permitiu realizar a adaptação transcultural para o Brasil do instrumento PAIR de avaliação da farmacoterapia na DRC. Os procedimentos adotados serviram para garantir a equivalência semântica, idiomática, experiencial e conceitual da versão brasileira em relação ao instrumento original, conforme recomendações metodológicas propostas por Guillemin et al. e Beaton et al. ${ }^{20,22-25}$.

A fase de análise das versões do PAIR pelo comitê de especialistas possibilitou corrigir imprecisões nas traduções e ajustar a redação dos itens ao contexto cultural brasileiro. Além disso, as sugestões dos profissionais atuantes no ambulatório de DRC e dos profissionais que aplicaram o pré-teste colaboraram para a obtenção do instrumento adaptado à realidade brasileira.

$\mathrm{Na}$ fase de pré-teste, a carta resposta enviada pela autora do artigo original foi essencial para compreensão do objetivo do termo utilizado em alguns PRMs "não tomar o medicamento apropriadamente". De acordo com a autora, a prescrição médica pode não incluir instruções completas para o paciente sobre a administração do medicamento, como horário, mesmo quando a dose e a freqüência estão devidamente prescritas. Um dos problemas desse fato é a interação medicamentosa, quando vários fármacos são administrados concomitantemente e um interfere no efeito do outro, ou, quando são administrados com o alimento, e este, interfere na sua absorção ${ }^{5}$. Nesse caso, o farmacêutico comunitário pode contribuir para a detecção de falhas e fornecer informações, indicando o melhor momento para a ingestão de cada medicamento, de acordo com as particularidades individuais.

Com relação aos PRMs rejeitados pelo comitê de especialistas, cinco foram da categoria "Aderência inadequada”. Isso porque essa categoria do PAIR avalia a aderência indiretamente baseado na quantidade de medicamentos fornecida ao paciente para uso em 90 dias, estando limitada a sistemas de informação, nacionais ou locais, que possuem registros sobre o fornecimento e dispensação de medicamentos. No Brasil não há controle eletrônico que permita monitorar a dispensação de medicamentos para pacientes renais crônicos. Dessa forma, não seria possível avaliar aderência ao tratamento a partir das informações do prontuário. Além disso, essa avaliação estaria suscetível a vieses, considerando que a aderência ao tratamento é um comportamento pessoal, que envolve o uso do medicamento de acordo com as recomendações médicas ${ }^{26}$. Estimar aderência com base em dados de fornecimento significa pressupor que tudo que foi fornecido foi ingerido, em doses prescritas, nos horários e durante o tempo prescrito, ou seja, significa ignorar uma série de elementos intermediários que não foram investigados ${ }^{26-28}$.

Outro PRM rejeitado foi da categoria "Hipoglicemia secundária à sulfoniluréia", que avalia reação adversa a Clorpropamida, uma sulfoniluréia de primeira geração que ainda é produzida e comercializada pela indústria farmacêutica. A rejeição deste PRM leva em consideração as recomendações da Sociedade Brasileira de Diabetes, segundo as quais, o uso das sulfoniluréias de primeira geração deve ser desencorajado por apresentarem efeitos colaterais expressivos, como ganho de peso e hipoglicemia, quando comparadas às outras sulfoniluréias ${ }^{29}$. Como esse fármaco não tem sido mais prescrito pelos médicos, e existe recomendação por consensos nacionais contrários ao seu uso em DRC, a avaliação deste PRM torna-se desnecessária.

Conforme exposto, a utilização de uma metodologia apropriada e aceita internacionalmente permitiu adaptar transculturalmente a versão inglesa do instrumento PAIR ao português brasileiro, rejeitando aqueles itens inapropriados em decorrência das diferenças no sistema de saúde entre os países. A exclusão da categoria "aderência inadequada" foi necessária em razão da ausência de um controle eletrônico de dispensação de fármacos no Brasil que não permitiria a real avaliação da adesão do paciente ao tratamento e, no caso do PRM "hipoglicemia secundária a sulfoniluréia", em razão da atualização de protocolos clínicos que não permitiria sua utilidade no check list.

Há algumas limitações atribuídas ao instrumento PAIR, uma vez que o mesmo foi desenvolvido, especificamente, para ser utilizado por farmacêuticos para a avaliação da farmacoterapia de indivíduos com DRC. A prevenção, detecção e gestão dos PRMs que constam nesse instrumen- 
to requerem informações claras e objetivas, que só poderão ser obtidas a partir de um prontuário completo, com dados do paciente, história clínica, registro cronológico dos resultados de exames laboratoriais, bem como de um atendimento médico ou farmacêutico que contemple essas informações. Dessa forma, cabe ressaltar que, a característica do serviço fornecido ao usuário irá impactar na aplicabilidade do instrumento. Contudo, essas limitações não inviabilizam o seu uso. Ao contrário, contribuem para que essas informações não sejam negligenciadas, pois consiste numa alternativa para ser utilizada de forma rápida e prática, a fim de sistematizar a avaliação da farmacoterapia e inserir o cuidado farmacêutico em serviços de nefrologia.

Além das limitações atribuídas ao instrumento, este estudo foi realizado em centro único, utilizando dados de uma amostra pequena, com o intuito de atender a metodologia proposta de adaptação transcultural. Dessa forma, a fim de verificar os resultados na prática assistencial a partir da aplicação desse instrumento, faz-se necessário conduzir um estudo em uma amostra maior e de caráter multicêntrico.

Espera-se assim, que a aplicação da versão do instrumento PAIR obtida neste trabalho, na prática clínica, permita identificar PRMs na farmacoterapia de pacientes renais crônicos em tratamento conservador e garantir segurança e resultados positivos no tratamento medicamentoso desses pacientes a partir de uma intervenção farmacêutica mais efetiva. Pela primeira vez que em nosso país, clínicos e nefrologistas poderão contar com um instrumento já amplamente utilizado em países desenvolvidos para detecção de PRMs em renais crônicos.

Todavia, estudos futuros deverão validar este instrumento para que elas possam ser utilizadas no contexto brasileiro.

\section{Colaboradores}

AB Marquito participou da revisão de literatura, coleta e análise de dados, redação e aprovação final do artigo. HS Pinheiro participou do desenho do estudo, análise dos dados, redação, revisão crítica e aprovação final do artigo. RB Paula participou do desenho do estudo, análise dos dados, redação, revisão crítica e aprovação final do artigo.

\section{Agradecimentos}

Os autores agradecem ao Centro Hiperdia Minas/ CEAE, à Fundação IMEPEN da cidade de Juiz de Fora/MG, ao Conselho Nacional de Desenvolvimento Científico e Tecnológico (CNPq), à Coordenação de Aperfeiçoamento de Pessoal de Nível Superior (CAPES) e à Fundação de Amparo à Pesquisa do Estado de Minas Gerais (FAPEMIG) por possibilitarem a realização da pesquisa. 


\section{Referências}

1. Romão Jr JE. Doença Renal Crônica: Definição, Epidemiologia e Classificação. J Bras Nefrol 2004; 26(3):1-3.

2. Sesso RC, Lopes AA, Thomé FS, Lugon JR, Martins CT. Inquérito Brasileiro de diálise crônica 2016. J Bras Nefrol 2017; 39(3):261-66.

3. Gouveia DSES, Bignelli AT, Hokazono SR, Danucalov I, Siemens TA, Meyer F, Santos LS; Martins ZCL, Mierzwa TC, Furquim R. Análise do impacto econômico entre as modalidades de terapia renal substitutiva. J Bras Nefrol 2017; 39(2):162-171.

4. Rocha CCT, Gomes ATL, Silva MF, Costa IKF, Mendes CKTT, Mendonça AEO, Torres GV. Hipertensos e diabéticos com insuficiência renal crônica no Brasil cadastrados no SIS/HIPERDIA. Rev Bras Hipertens 2015; 22(1):27-32.

5. Marquito AB, Fernandes NMS, Colugnati FAB, De Paula RB. Interações medicamentosas potenciais em pacientes com doença renal crônica. J Bras Nefrol 2014; 36(1):26-34.

6. Cardone KE, Bacchus S, Assimon MM, Pai AB, Manley HJ. Medication-related problems in CKD. $A d v$ Chronic Kidney Dis 2010; 17(5):404-412.

7. Mason NA. Polypharmacy and medication-related complications in the chronic kidney disease patient. Curr Opin Nephrol Hypertens 2011; 20(5):492-497.

8. Hernández DS, Castro MMS, Dáder MJF. Método Dáder. Manual de Seguimento Farmacoterapêutico, 2011, versão em português do Brasil. Granada: Grupo de Investigación en Atención Farmacéutica (CTS-131), Universidad de Granada; 2011.

9. Ivama AM, Noblat L, Castro MS, Oliveira NVBV, Jaramillo NM, Rech N. Proposta - Consenso brasileiro de atenção farmacêutica. Brasília: OPAS; 2002.

10. Huri HZ, Wee HF. Drug related problems in type 2 diabetes patients with hypertension: a cross-sectional retrospective study. BMC Endocr Disord 2013; 13:2.

11. Gastelurrutia P, Benrimoj SI, Espejo J, Tuneu L, Mangues MA, Bayes-Genis A. Negative Clinical Outcomes Associated With Drug-Related Problems in Heart Failure (HF) Outpatients: Impact of a Pharmacist in a Multidisciplinary HF Clinic. J Card Fail 2011; 17(3):217-223.

12. Quintana-Bárcena P, Lord A, Lizotte A, Berbiche D, Lalonde L. Prevalence and Management of Drug-Related Problems in Chronic Kidney Disease Patients by Severity Level: A Subanalysis of a Cluster Randomized Controlled Trial in Community Pharmacies. JMCP 2018; 24(2):173-181.

13. Desrochers JF, Lemieux JP, Morin-Bélanger C, Paradis FS, Lord A, Bell R, Berbiche D, Bárcena PQ, Séguin NC, Desforges K, Legris MẼ, Sauvé P, Lalonde L. Development and Validation of the PAIR (Pharmacotherapy Assessment in Chronic Renal Disease) Criteria to Assess Medication Safety and Use Issues in Patients With CKD. Am J Kidney Dis 2011; 58(4):527-535.

14. Raymond CB, Wazny LD, Sood AR. Standards of Clinical Practice for Renal Pharmacists. Can J Hosp Pharm 2013; 66(6):369-374
15. St Peter WL, Wazny LD, Patel UD. New models of chronic kidney disease care including pharmacists: improving medication reconciliation and medication management. Curr Opin Nephrol Hypertens 2013; 22(6):656-662.

16. Smits KPJ, Sidorenkov G, Bilo HJG, Bouma M, Navis GJ, Denig P. Process quality indicators for chronic kidney disease risk management: a systematic literature review. Int J Clin Pract 2016; 70(10):861-869.

17. Grabe DW, Cardone KE. Altered pharmacology and pill burden in older adults: A balancing act. In: Misra M. Dialysis in older adults: A Clinical Handbook. New York: Springer; 2016. p. 123-146.

18. Joosten H, Drion I, Boogerd KJ, van der Pijl EV, Slingerland RJ, Slaets JPJ, Jansen TJ, Schwantje O, Gans $\mathrm{RO}$, Bilo HJ. Optimising drug prescribing and dispensing in subjects at risk for drug errors due to renal impairment: improving drug safety in primary healthcare by low eGFR alerts. BMJ Open 2013; 3:1.

19. Wood GL, Haber J. Desenhos não-experimentais. In Wood GL, Haber J, organizadores. Pesquisa em Enfermagem: métodos, avaliação crítica e utilização. Rio de Janeiro: Guanabara Koogan; 2001.p. 110-121.

20. Guillemin F, Bombardier C, Beaton DE. Cross-cultural adaptation of health- related quality of life measures: literature review and proposed guidelines. J Clin Epidemiol 1993; 46(12):1417-1432.

21. Fitch K, Bernstein SJ, Aguilar MD, Burnand B, LaCalle JR, Lazaro P, Loo MVH, McDonnell J, Vader J, Kahan JP. The RAND/UCLA Appropriateness Method User's Manual. Santa Monica: RAND Corporation; 2001.

22. Herdman M, Fox-Rushby J, Badia X. Equivalence and the translation and adaptation of health-related quality of life questionnaires. Qual Life Res 1997; 6:237247.

23. Bullinger $\mathrm{M}$, Alonso $\mathrm{A}$, Apolone $\mathrm{G}$, Leplège $\mathrm{A}$, Sullivan M, Wood-Dauphinee S, Gandek B, Wagner A, Aaronson N, Bech P, Fukuhara S, Kaasa S, Ware JE Jr. Translating health status questionnaires and evaluating their quality: the IQOLA approach. J Clin Epidemiol 1998; 51:913-923.

24. Beaton DE, Bombardier C, Guillemin F, Ferraz M B. Guidelines for the Process of Cross-Cultural Adaptation of Self-Report Measures. SPINE 2000; 25(24):3186-3191.

25. Beaton DE, Bombardier C, Guillemin F, Ferraz M B. Recommendations for the cross-cultural adaptation of health status measures. New York: American Academy of Orthopaedic Surgeons; 2002.

26. Magacho EJC, Ribeiro LC, Chaoubah A, Bastos MG. Adherence to drug therapy in kidney disease. Braz J Med Biol Res 2011; 44(3):258-262.

27. Marsicano EO, Fernandes NS, Colugnati F, Grincenkov FRS, Fernandes NMS; De Geest, S; Sanders-Pinheiro, H. Transcultural adaptation and initial validation of Brazilian-Portuguese version of the Basel assessment of adherence to immunosuppressive medications scale (BAASIS) in kidney transplants. BMC Nephrology 2013; 14:108. 
28. Lima-Dellamora EC, Osorio-de-Castro CGS, Madruga LGSL, Azeredo TB. Utilização de registros de dispensação de medicamentos na mensuração da adesão: revisão crítica da literatura. Cad Saúde Pública 2017; 33(3):e00136216.

29. Sociedade Brasileira de Diabetes. VI Diretrizes da Sociedade Brasileira de Diabetes: 2015-2016. São Paulo: A.C. Farmacêutica; 2016.

Artigo apresentado em 24/07/2018

Aprovado em 09/02/2019

Versão final apresentada em 11/02/2019 